\title{
Capacity Estimation for a DS-CDMA System in Nakagami-m Fading
}

\author{
PANAGIOTIS VARZAKAS \\ Department of Informatics and Telecommunications \\ University of Thessaly \\ 3o Km Old Road Lamia-Athens \\ GREECE
}

\begin{abstract}
In this paper, a novel closed-form expression of the Shannon average channel capacity per user for direct- sequence code-division multiple access (DS-CDMA) systems, operating in Nakagami- $m$ fading, with optimum RAKE reception, is obtained. Numerical results are also presented to illustrate the proposed mathematical analysis and to point out the effect of the fading severity on the user's average channel capacity.
\end{abstract}

Key-Words: DS-CDMA systems, Channel capacity, Nakagami-m fading.

Received: July 15, 2021. Revised: January 11, 2022. Accepted: February 10, 2022. Published: March 3, 2022.

\section{Introduction}

RAKE reception, with maximal ratio combining (MRC), is an effective way to anticipate multipath signal fading, due to its ability to resolve additional multipaths, resulting in an increased diversity gain, [1]. Moreover, channel capacity, in the Shannon sense, is a significant criterion for the design and the performance evaluation of digital communication systems, [2]. Thus, an estimation of the average channel capacity based on optimal rate adaptation to channel fading and constant transmit power could indeed provide the maximum transmission rates, if channel side information were available at the receiver.

In this paper, a closed-form analytical expression for the Shannon channel capacity per user in direct-sequence code-division multiple access (DS-CDMA) systems with MRC RAKE reception is obtained, extending the results of [3] and [4] for the important Nakagami- $m$ fading channel model. Numerical results are also presented to illustrate the proposed mathematical analysis. In these results, it is pointed out the effect of the fading severity on the user's capacity and a comparison with the capacity of the additive white Gaussian noise (AWGN) channel is also given.

\section{System and channel model}

We consider a non-cooperative DS-CDMA wireless system that consists of $K$ simultaneous users, each transmitting with the same average power. Bandwidth spreading is accomplished at the transmitter by multiplying the information data by a broadband code sequence. Each user transmits a signal of bandwidth $W_{s s}$ after spreading the actual signal bandwidth $W$ by the system's processing gain $G_{p}=\frac{W_{s s}}{W}$.
The channel capacity of each user of such a system, i.e. a single user's conditional channel capacity in the Shannon sense, called hereafter "channel capacity per user", will clearly depend on the level of cooperation among the $K>1$ users or, equivalently, on the multiple-access interference (MAI) power. This channel capacity per user will be given by the Shannon-Hartley theorem when arbitrarily complex coding and delay is applied, [5], while the total MAI power, caused by even a small number of interfering users, will tend to be Gaussian distributed, [6].

Each user's RAKE receiver has $L$ taps corresponding to $L$ resolvable signal paths of the multipath tapped delay line channel model, whose fading amplitudes and phases are perfectly known. $L=\left[T_{m} W_{s s}\right]+1$, where $T_{m}$ is the total multipath delay spread of the Nakagami- $m$ fading channel on the condition that the transmitted signal bandwidth $W_{s s}$ is much greater than the coherence bandwidth $W_{c o h}$ of the fading channel, with $[x]$ be the maximum integer less than or equal to $x$.

\subsection{AWGN channel}

For a single user transmitting signal, of bandwidth $W$, in the AWGN channel, the received signal-to-noise ratio (SNR) is $Z=\frac{P}{N_{0} W}$ where $N_{0}$ is the double-sided noise power spectral density and $P$ is the received power. When this user operates in the considered DS-CDMA system, his signal will clearly be affected by the Gaussian distributed MAI of all the other $(K-1)$ simultaneous users, the power of its is $P_{M A I}=\frac{K-1}{P}$. The received spread signal-to-interference-plus-noise power ratio (SINR) (prior despreading) will then be 
$Z_{s s}=\frac{Z}{\left(G_{p}+(K-1) \cdot Z\right)}$. Accordingly, the channel capacity per user is [2, Eq. $(15-2-8)]$ :

$$
\mathrm{C}_{\text {user }}=W_{\mathrm{ss}} \cdot \log _{2}\left(1+Z_{s s}\right)
$$

and the spread channel incorporated will appear with a total channel capacity $C_{t}$, available to all users, being equal to the sum of the corresponding individual rates, i.e. $C_{t}=K \cdot C_{u s e r}$.

\subsection{Nakagami- $m$ fading channel}

Assuming independent and identical distributed (i.i.d.) input paths, the probability density function (pdf) of the instantaneous received signal-to-interference-plus-noise ratio (SINR), $\gamma_{i, s s}$, in the $l$ th, $l=1, \ldots, L$, branch of the $i$ th, MRC RAKE receiver is a Gamma distribution with two degrees of freedom:

$$
p\left(\gamma_{l, i, s s}\right)=\left(\frac{m}{\bar{\gamma}_{l, i, s s}}\right)^{m} \cdot \frac{\gamma^{m}{ }_{l, i, s s}}{\Gamma(m)} \cdot e^{-} \frac{\gamma_{l, i, s s}}{\bar{\gamma}_{l, i, s s}}
$$

where $\bar{\gamma}_{l, i, s s}$ is the corresponding average received SINR, resulting from the signals of all transmitting users, $\Gamma(x)$ is the gamma function defined as $\Gamma(x)=\int_{0}^{\infty} e^{-t} t^{x-1} d t$ and $m$ is the Nakagami- $m$ fading parameter $(m \geq 0.5)$. When $m=1$ the fading envelopes are Rayleigh distributed and (2) reduces to the exponential distribution. The statistics of each interfering signal in (2) need not considered separately since, either the total interference power at the RAKE receiver output, or the MAI from the $(K-1)$ other users prior despreading, even for a small number of users, tends to be Gaussian distributed [6], and thus, it can directly be incorporated in the Shannon formula regardless the interference statistics.

When the $K$ users are simultaneously transmitting over a Nakagami- $m$ fading channel, the pdf of the combined SNR, $\gamma_{m r c}$, assuming equal path strengths, $\bar{\gamma}_{l, i, s s}=\bar{\gamma}_{s s}$, at the MRC RAKE receiver output, will be given by [1]:

$$
p\left(\gamma_{m r c}\right)=\left(\frac{m}{\bar{\gamma}_{s s}}\right)^{m L} \cdot \frac{\gamma_{m r c}^{m L-1}}{\Gamma(m L)} \cdot e^{-\frac{\gamma_{m r c}}{\gamma_{s s}}}
$$

where the corresponding average received SINR, $\bar{\gamma}_{m r c}$, will be:

$$
\bar{\gamma}_{m r c}=L \cdot \bar{\gamma}_{s s}
$$

The average total channel capacity, $\left\langle C_{t}\right\rangle$, will be given by averaging $C_{t}$ over the pdf of the combined SNR, $\gamma_{m r c}$, at the RAKE receiver's output [7], i.e.

$$
\left\langle C_{t}\right\rangle=W_{s s} \cdot \int_{0}^{\infty} \log _{2}\left(1+\gamma_{m r c}\right) \cdot p\left(\gamma_{m r c}\right) d \gamma_{m r c}
$$

By replacing (3) and (4) in the above equation and using [8, Eq. (32)], the average channel capacity per user, normalized over the signal bandwidth, will be given by a closed-form expression as:

$$
\begin{aligned}
& \frac{\left\langle C_{t}\right\rangle}{W}=\frac{G_{p}}{K} \cdot \frac{1}{\Gamma(m L)} \cdot\left\{\frac{m L\left[G_{p}+(K-1) Z\right]}{Z}\right\}^{m L} . \\
& I_{m L}\left\{\frac{m L\left[G_{p}+(K-1) Z\right]}{Z}\right\}
\end{aligned}
$$

where $I_{n}(\mu)$ is defined as $I_{n}(\mu)=(n-1) ! e^{\mu} \cdot \sum_{k=1}^{n} \Gamma(-n+k, \mu) \cdot \mu^{-k}$ and $\Gamma(\alpha, x)$ is the incomplete gamma function, $\Gamma(\alpha, x)=\int_{x}^{\infty} e^{-t} t^{\alpha-1} d t$. It is interesting to note that, for $\quad K=1, \quad m=1 \quad$ and $\quad G_{p}=1$ $(L=1)$, (6) directly leads to the expression of the average channel capacity of a single user transmitting, without spreading the signal bandwidth $W$ as it first appeared in [7]. In addition, considering a DS-CDMA system, with a transmitted signal bandwidth $W_{\mathrm{ss}}$ that tends to infinity, i.e. with $G_{p} \rightarrow \infty$, the average channel capacity per user $\left\langle C_{u s e r}\right\rangle$ will tend to the capacity of a AWGN channel bandlimited to $W$, i.e. $\left\langle C_{\text {user }}\right\rangle=W \cdot \log _{2}(1+Z)$, since in this case, the pdf of $\gamma_{m r c}$ in (3) will be given by the delta-function [7].

\section{Numerical results}

By evaluating (6), numerical results are presented in Figs. 1 and 2. In both figures a typical urban area with $T_{m}=1.5 \mu \mathrm{s}$ is considered, with $K=10$ simultaneously transmitting active users, $G_{p}=100$ and $W=20 \mathrm{KHz}$. In Fig. $1,\left\langle C_{\text {user }}\right\rangle / W$ is plotted as function of $Z$ for RAKE reception and $m=0.5,1,2$ and 4. In the same figure, using (1), the corresponding curve for the AWGN channel is also plotted for comparison reasons. As it is clear 
$\left\langle C_{\text {user }}\right\rangle / W$ increases with $Z$ and a floor is observed due to MAI. For a fixed $Z,\left\langle C_{\text {user }}\right\rangle / W$ increases as the fading severity decreases (i.e. $m$ increases) and it gets very close to the corresponding curve of the AWGN for $m=4$.

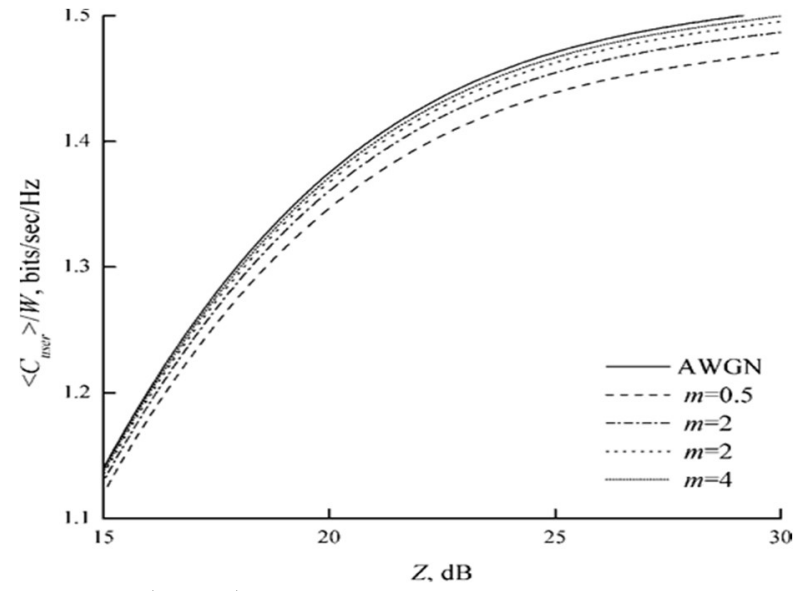

Fig. $1\left\langle C_{\text {user }}\right\rangle / W$ versus $\mathrm{Z}$ for typical urban area with $T_{m}=1.5 \mu \mathrm{s}, K=10$ users, $G_{p}=100, W=20 \mathrm{KHz}$ and several values of $m$.

In Fig. 2, $\left\langle C_{\text {user }}\right\rangle / W$ is plotted as function of $m$ for RAKE reception and $Z=20,25$ and $30 \mathrm{~dB}$. As $m$ increases, $\left\langle C_{\text {user }}\right\rangle / W$ increases, as it was also observed in Fig. 1.

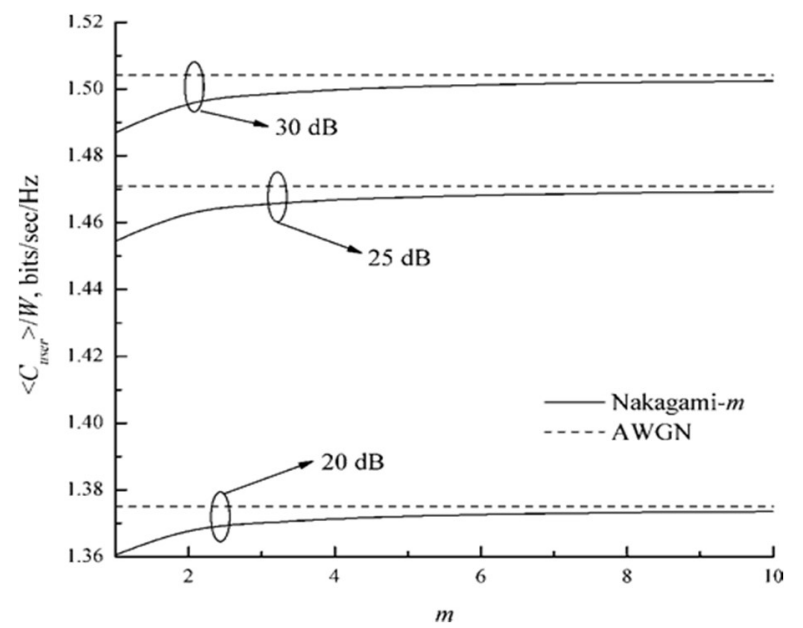

Fig. $2\left\langle C_{\text {user }}\right\rangle / W$ versus $m$ for typical urban area with $T_{m}=1.5 \mu \mathrm{s}, K=10$ users, $G_{p}=100, W=20 \mathrm{KHz}$ and several values of $Z$.

\section{Conclusions}

In this paper, an analytical closed-form expression for the average channel capacity per user, in the Shannon sense, of a non-cooperative DS-CDMA system over a Nakagami- $m$ fading channel under MRC RAKE reception has been derived. Numerical results have pointed the effect of the fading severity and MAI on the system's channel capacity.

\section{References:}

[1] M.K.Simon and M.S.Alouini, Digital communication over fading channels, John Wiley, 2nd edn, 2005.

[2] J.G.Proakis and M.Salehi, Digital Communications, 5th edn, McGraw-Hill, 2008.

[3] P.Varzakas and G.S.Tombras, Average channel capacity for RAKE receivers, IEE Electronics Letters, Vol.38, No.10, 2002, pp.475-476.

[4] P.Varzakas, Average Channel Capacity for Rayleigh Fading Spread Spectrum MIMO systems, International Journal of Communication Systems, Vol. 19, No.10, 2006, pp.1081-1087.

[5] C.E.Shannon, Communication in the presence of noise, Proceedings of IRE, Vol.37, 1949, pp.1021.

[6] K.S.Gilhousen, I.M.Jacobs, R.Padovani, A.Viterbi L.A.Weaver and C.E.Wheatley, On the capacity of a cellular CDMA system, IEEE Transactions on Vehicular Technology, Vol.40, 1991, pp.303-312.

[7] W.C.Y.Lee, Estimate of channel capacity in Rayleigh fading environment, Vol. 39, 1990, pp. 187-189.

[8] M.S.Alouini and A.J.Goldsmith, Capacity of Rayleigh fading channels under different adaptive transmission and diversity-combining techniques, IEEE Transactions on Vehicular Technology, Vol.48, No.4, 1999, pp.1165-1181.

\section{Creative Commons Attribution License 4.0 (Attribution 4.0 International, CC BY 4.0)}

This article is published under the terms of the Creative Commons Attribution License 4.0 https://creativecommons.org/licenses/by/4.0/deed.en_US 